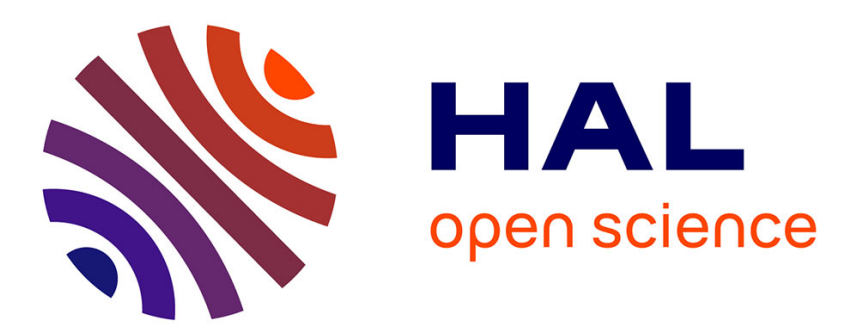

\title{
Effects of porous confinement on the structural properties of the Gaussian core model
}

Dieter Franz Schwanzer, Daniele Coslovich, Jan Kurzidim, Gerhard Kahl

\section{To cite this version:}

Dieter Franz Schwanzer, Daniele Coslovich, Jan Kurzidim, Gerhard Kahl. Effects of porous confinement on the structural properties of the Gaussian core model. Molecular Physics, 2009, 107 (04-06), pp.433-441. 10.1080/00268970902845321 . hal-00513268

\section{HAL Id: hal-00513268 \\ https://hal.science/hal-00513268}

Submitted on 1 Sep 2010

HAL is a multi-disciplinary open access archive for the deposit and dissemination of scientific research documents, whether they are published or not. The documents may come from teaching and research institutions in France or abroad, or from public or private research centers.
L'archive ouverte pluridisciplinaire HAL, est destinée au dépôt et à la diffusion de documents scientifiques de niveau recherche, publiés ou non, émanant des établissements d'enseignement et de recherche français ou étrangers, des laboratoires publics ou privés. 


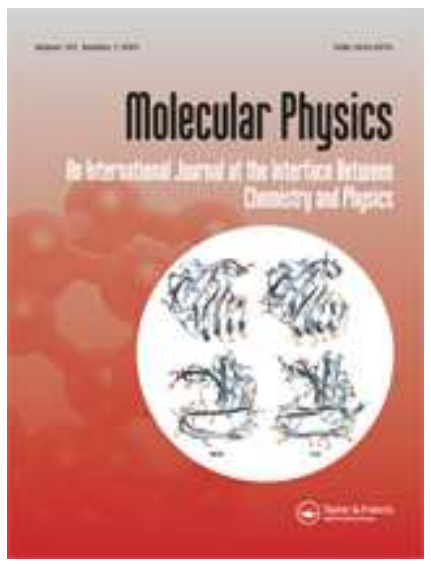

\section{Effects of porous confinement on the structural properties of the Gaussian core model}

\begin{tabular}{|r|l|}
\hline Journal: & Molecular Physics \\
\hline Manuscript ID: & TMPH-2009-0017.R1 \\
\hline Manuscript Type: & Special Issue Paper - Dr. Jean-Jacques Weis \\
\hline Author: & 13-Feb-2009 \\
\hline Complete List of Authors: & $\begin{array}{l}\text { Schwanzer, Dieter; TU Wien, Institute for Theoretical Physics and } \\
\text { Centre for Computational Material Science } \\
\text { Coslovich, Daniele; TU Wien, Institute for Theoretical Physics and } \\
\text { Centre for Computational Material Science } \\
\text { Kurzidim, Jan; TU Wien, Institute for Theoretical Physics and Centre } \\
\text { for Computational Material Science } \\
\text { Kahl, Gerhard; TU Wien, Institut für Theoretische Physik }\end{array}$ \\
\hline Keywords: & $\begin{array}{l}\text { liquids, porous media, soft matter, replica Ornstein-Zernike } \\
\text { equations, Monte Carlo simulation }\end{array}$ \\
\hline $\begin{array}{l}\text { Note: The following files were submitted by the author for peer review, but cannot be converted } \\
\text { to PDF. You must view these files (e.g. movies) online. }\end{array}$ \\
\hline Effects_of_porous_confinement.tex \\
\hline \hline
\end{tabular}

\section{S ScholaroNE Manuscript Central}


Molecular Physics

Vol. 00, No. 00, 10 May 2008, 1-19

\title{
RESEARCH ARTICLE
}

\section{Effects of porous confinement on the structural properties of the \\ Gaussian core model}

\author{
Dieter F. Schwanzer*, Daniele Coslovich, Jan Kurzidim, Gerhard Kahl \\ Institute for Theoretical Physics and Centre for Computational Material Science (CMS), \\ TU Wien, Wiedner Hauptstraße 8-10, A-1040 Vienna, Austria;
}

\begin{abstract}
We have investigated the structural properties of a fluid in which particles, interacting via soft potentials, are imbibed into a disordered porous structure built up by soft particles. Using a Gaussian potential for all interactions involved, we determine via Monte Carlo simulations and integral-equation theory the fluid-fluid, fluid-matrix, connected, and blocked structure factors. Within the explored range of state parameters, the fluid-fluid structure factors display a distinct pre-peak, which we identify as a fingerprint of the structure of the matrix. We show that this feature at low wave-vectors arises from contributions of blocked correlations to the fluid-fluid structure factor. We argue that a similar feature may be found also in systems in which particles interact via harshly repulsive potentials. On the other hand, the variation of the main peak of the fluid-fluid structure factor resembles the behaviour of the equilibrium Gaussian core model fluid. In particular, the height of the main peak changes non-monotonically with increasing fluid density at fixed matrix density. Finally, we analyse the effect of a mismatch between the temperature of the fluid and the temperature of the matrix on the structural properties of the system.
\end{abstract}

Keywords: liquids, porous media, soft matter, replica Ornstein-Zernike equations, Monte Carlo simulation

*Corresponding author. Email: schwanzer@cmt.tuwien.ac.at

ISSN: 00268976 print/ISSN 13623028 online

(C) 2008 Taylor \& Francis

DOI: $10.1080 / 0026897 Y Y x x x x x x x x$

http://www.informaworld.com 


\section{Introduction}

When a fluid is brought into contact with a disordered porous matrix, its physical properties change drastically. A large number of experimental and theoretical studies performed during past years provide unambiguous evidence for this phenomenon. This field of research is, however, not only of pure academic interest, but rather has high technological relevance. For an overview of the vast literature available in this field we refer to Refs. [1-3]. Most of the experimental investigations carried out so far were dedicated to so-called 'hard' systems, where typically atomic or molecular fluids (as, for instance, ${ }^{4} \mathrm{He}, \mathrm{N}_{2}$, or ${ }^{3} \mathrm{He}-{ }^{4} \mathrm{He}$ mixtures) were brought into contact with matrices such as Vycor, aerogel, or silica gels. Likewise, most of the theoretical investigations were dedicated to systems where particles interact via a harsh repulsion plus some adjacent (attractive) potential tail: among those one finds simple fluids (e.g., [2, 4-6] and references therein), binary mixtures [7-9], or fluids with orientational degrees of freedom [10-14].

The rapidly growing interest in soft matter systems during recent years stimulates the obvious extension to investigate how the properties of a soft system (typically, a colloidal dispersion) change when it is brought into contact with a (soft) porous matrix. Only a few contributions have been dedicated up to now to this problem (e.g., $[15,16])$. Recent experimental and theoretical progress in soft matter physics provide a sufficient amount of motivation to extend investigations also to soft systems that are in contact with a disordered porous matrix: on the experimental side, the considerably larger size of the particles (typically ranging from a few nm to $\mu \mathrm{m}$ ) allows a more convenient observation of the system by using techniques such as video [17] and/or confocal [18] microscopy. In addition, optical tweezers [19] offer the possibility to fix particles in deliberately chosen positions, tailoring thereby a suitable matrix configuration. From the theoretical point of view, the description of particle interactions in soft systems via effective potentials [20] has reached nowadays a very high level of sophistication, so that even for colloidal particles with a complex internal architecture highly reliable effective interactions can be deduced via suitable averaging procedures over the huge number of internal degrees of freedom. Finally, theoretical frameworks have been proposed to consider more realistic models for matrix representations [21-26].

This contribution is dedicated to an investigation of how the presence of a disordered matrix, built up by soft particles, influences the structural properties of a 
soft fluid that is imbibed into this porous structure. Such a system is commonly referred to as a quenched-annealed (QA) system, where the matrix configurations are quenched from an equilibrium fluid and the fluid component is subsequently allowed to equilibrate in it. Since we aim at systematic investigations of structural trends, we have restricted ourselves to one of the standard models in soft matter physics, i.e., the Gaussian core model (GCM). This model, originally introduced several years ago $[27,28]$ in a different context, has meanwhile turned out to be a reasonably reliable model system for colloidal dispersions, such as polymers, dendrimers, or microgels. In this contribution we have used the Gaussian potential for all interactions involved, namely the matrix-matrix, the matrix-fluid, and the fluidfluid potential. The structural properties of the system were obtained via standard Monte Carlo simulations and via integral-equation theory. In the latter case our investigations are based on the well-established replica Ornstein-Zernike (ROZ) formalism [29, 30], a framework that is known to be highly successful in describing the structural and thermodynamic properties of fluids confined in disordered porous structures. The first part of the investigation is dedicated to the influence of the structure of the matrix on the structural properties of the fluid, identifying thereby fingerprints of the matrix-matrix correlations in the fluid-fluid structure factor. These findings are interpreted on a more profound level in the second part of the study, where we focus on the so-called blocked and connected contributions to the fluid-fluid correlation functions. Finally, we study the influence of a mismatch in the temperature of the quenched matrix configuration and of the equilibrated fluid on the structural properties.

This contribution is organised as follows: in the subsequent section we present our model and the theoretical methods that we use to calculate the structural properties of our system. In Section 3 we discuss our results and then close the contribution with concluding remarks.

\section{Model and Methods}

The matrix of our QA system is generated from a configuration of an equilibrated fluid of matrix density $\rho_{\mathrm{m}}$ and matrix temperature $T_{\mathrm{m}}$ by fixing at some instant the positions of the particles. The annealed component of fluid number density $\rho_{\mathrm{f}}$ is imbibed into the system and is allowed to equilibrate at a temperature $T_{\mathrm{f}}$.

The interactions between the different types of particles in the system, charac- 


\section{Molecular Physics}

terised by index combinations 'mm', 'fm', and 'ff' ('f' standing for the fluid and 'm' for the matrix) are parametrised by the Gaussian core model potential,

$$
\Phi_{\mathrm{GCM}}(r)=\epsilon e^{-(r / \sigma)^{2}}
$$

where $\sigma$ and $\epsilon$ are length- and energy-scales used in the following as units of length and energy, respectively. The GCM, first proposed by Flory and Kriegbaum [27] and Stillinger [28], can be considered as a simple, but reasonably effective model potential between the centres of mass of two linear polymer chains. Meanwhile it has become one of the standard models in soft matter physics. One reason for our choice of this particular interaction is the fact that the equilibrium phase diagram of the GCM is known with high accuracy [31, 32].

\subsection{Replica Ornstein-Zernike Equations}

A successful theoretical approach to describe the structural and thermodynamic properties of QA systems was pioneered by Madden and Glandt [29] and later by Given and Stell [30]. The properties of the annealed component that equilibrates in the presence of a matrix are calculated in a double averaging procedure: first, over the degrees of freedom of the fluid particles for a given matrix configuration, and, second, over all possible matrix configurations. This averaging can conveniently be effectuated using the replica trick [33]. Starting from the Ornstein-Zernike equations of an equilibrated $(s+1)$ component system, which consists of the matrix and $s$ non-interacting copies (replicas) of the fluid, one arrives, after taking the limit $s \rightarrow 0$, at the replica Ornstein-Zernike equations $[29,30]$

$$
\begin{aligned}
h_{\mathrm{mm}}(r) & =c_{\mathrm{mm}}(r)+\rho_{\mathrm{m}} c_{\mathrm{mm}}(r) \otimes h_{\mathrm{mm}}(r) \\
h_{\mathrm{fm}}(r) & =c_{\mathrm{fm}}(r)+\rho_{\mathrm{m}} c_{\mathrm{fm}}(r) \otimes h_{\mathrm{mm}}(r)+\rho_{\mathrm{f}} c_{\mathrm{c}}(r) \otimes h_{\mathrm{fm}}(r) \\
h_{\mathrm{ff}}(r) & =c_{\mathrm{ff}}(r)+\rho_{\mathrm{m}} c_{\mathrm{fm}}(r) \otimes h_{\mathrm{fm}}(r)+\rho_{\mathrm{f}} c_{\mathrm{c}}(r) \otimes h_{\mathrm{c}}(r) \\
h_{\mathrm{rr}}(r) & \left.=c_{\mathrm{rr}^{\prime}}(r)+\rho_{\mathrm{m}} c_{\mathrm{fm}}(r) \otimes h_{\mathrm{fm}}(r)+\rho_{\mathrm{f}} c_{\mathrm{ff}}(r) \otimes h_{\mathrm{rr}^{\prime}}(r)+\rho_{\mathrm{f}} c_{\mathrm{rr}^{\prime}}(r) \otimes h_{\mathrm{ff}}(r)-2 \rho_{\mathrm{f}} c_{\mathrm{rr}^{\prime}}(r) \otimes h_{\mathrm{rr}^{\prime}}((55))\right)
\end{aligned}
$$

where the indices ' $r$ ' and ' $r$ ' stand for distinct replica components. The $h_{i j}(r)$ and the $c_{i j}(r)$ are the total and the direct correlation functions, respectively. Further we introduce the connected correlation functions $h_{\mathrm{c}}(r)=h_{\mathrm{ff}}(r)-h_{\mathrm{rr}^{\prime}}(r)$ and $c_{\mathrm{c}}(r)=$ 
$c_{\mathrm{ff}}(r)-c_{\mathrm{rr}}(r)$ as well as the blocked correlation functions $h_{\mathrm{b}}(r)=h_{\mathrm{ff}}(r)-h_{\mathrm{c}}(r)$ and $c_{\mathrm{b}}(r)=c_{\mathrm{ff}}(r)-c_{\mathrm{c}}(r)$. In the relations above, the symbol $\otimes$ stands for a convolution integral. Note that the first of the ROZ equation [Equation (2)] is decoupled from the remaining three relations, which, in turn, form a set of coupled integral-equations that has to be solved - similar as in the equilibrated case [34] in combination with a suitable closure relation that relates the set of correlation functions with the corresponding interactions.

Justified by the general observation that for bounded, purely repulsive interactions the hypernetted chain (HNC) closure is considered to be a highly appropriate choice [31, 35], we use this particular relation as a complement to the ROZ equations. The HNC closure reads

$$
h_{i j}(r)+1=\exp \left[-\beta_{\nu} \Phi_{i j}(r)+h_{i j}(r)-c_{i j}(r)\right]
$$

where ' $i j$ ' stands for the different index combinations introduced above and ' $\nu$ ' stands for the index $\mathrm{m}$ in case $i j=\mathrm{mm}$ and $\mathrm{f}$ in all other cases. Note that $\Phi_{\mathrm{rr}^{\prime}}(r) \equiv$ 0 and $\Phi_{i j}(r)=\Phi_{\mathrm{GCM}}(r)$ otherwise. Moreover, the formalism allows to take into account independently both the temperature of the matrix, $\beta_{\mathrm{m}}=\left(k_{\mathrm{B}} T_{\mathrm{m}}\right)^{-1}$, as well as the temperature of the fluid, $\beta_{\mathrm{f}}=\left(k_{\mathrm{B}} T_{\mathrm{f}}\right)^{-1}$. The numerical solution of the ROZ equations [Equations (2)-(5)] in combination with the closure relations [Equation (6)] has been realised via a standard iterative procedure for solving coupled integral-equations.

For the present investigations the structure factors are of particular relevance. They are defined as functions of the wave number $k$ as follows:

$$
\begin{gathered}
S_{\mathrm{mm}}(k)=1+\rho_{\mathrm{m}} \tilde{h}_{\mathrm{mm}}(k) \\
S_{\mathrm{fm}}(k)=\sqrt{\rho_{\mathrm{m}} \rho_{\mathrm{f}}} \tilde{h}_{\mathrm{fm}}(k) \\
S_{\mathrm{ff}}(k)=1+\rho_{\mathrm{f}} \tilde{h}_{\mathrm{ff}}(k) \\
S_{\mathrm{c}}(k)=1+\rho_{\mathrm{f}} \tilde{h}_{\mathrm{c}}(k) \\
S_{\mathrm{b}}(k)=S_{\mathrm{ff}}(k)-S_{\mathrm{c}}(k),
\end{gathered}
$$

where the tilde denotes the Fourier-transform of the respective total correlation function. 


\section{Molecular Physics}

\subsection{Monte Carlo Simulations}

The theoretical data are complemented by extensive Monte Carlo (MC) simulations where the above mentioned double average has been carried out explicitly. In the practical realisation of this recipe we have first generated a matrix configuration from an independent simulation run, fixing the positions of the particles at some arbitrarily chosen instant once the system has equilibrated at temperature $T_{\mathrm{m}}$. Then, the fluid particles are brought into contact with this porous matrix configuration and the subsequent simulation of the system is carried out at a temperature $T_{\mathrm{f}}$.

The simulations were performed in a cubic box of side length $L=10$, featuring periodic boundary conditions. Typically $50 \leq N_{\mathrm{m}} \leq 300$ matrix particles and $50 \leq N_{\mathrm{f}} \leq 500$ fluid particles were considered. To speed up the simulation we have used the lattice Monte Carlo technique introduced by Panagiotopoulos [36]. The simulation-box was sub-divided into 256 lattice positions in each direction, forming a lattice of $256^{3}$ possible particle positions. We point out that no change in the structural properties could be observed using a higher number of lattice positions. To increase the efficiency of the simulation even further we also used the cell-list method [37]. Simulations extended over 1,000,000 MC sweeps. Correlation functions were obtained by averaging over at least 10,000 fluid configurations and over 10-40 independent matrix configurations.

Following the formalism of Krakoviack [38], the structure factors introduced above were calculated directly via the Fourier-components of the respective one particle densities as follows: introducing

$$
\rho_{\mathrm{m}}(\mathbf{k})=\sum_{j=1}^{N_{\mathrm{m}}} e^{i \mathbf{k} \cdot \mathbf{s}_{j}} \quad \rho_{\mathrm{f}}(\mathbf{k})=\sum_{j=1}^{N_{\mathrm{f}}} e^{i \mathbf{k} \cdot \mathbf{r}_{j}}
$$

where the $\mathbf{s}_{j}$ are the positions of the immobile matrix particles and the $\mathbf{r}_{j}$ are the positions of the fluid particles, the expressions for the structure factors read

$$
\begin{gathered}
S_{\mathrm{ff}}(k)=\frac{1}{N_{\mathrm{f}}} \overline{\left\langle\rho_{\mathrm{f}}(\mathbf{k}) \rho_{\mathrm{f}}(-\mathbf{k})\right\rangle} \\
S_{\mathrm{fm}}(k)=\frac{1}{\sqrt{N_{\mathrm{f}} N_{\mathrm{m}}}} \overline{\left\langle\rho_{\mathrm{f}}(\mathbf{k}) \rho_{\mathrm{m}}(-\mathbf{k})\right\rangle}
\end{gathered}
$$




$$
\begin{gathered}
S_{\mathrm{b}}(k)=\frac{1}{N_{\mathrm{f}}} \overline{\left\langle\rho_{\mathrm{f}}(\mathbf{k})\right\rangle\left\langle\rho_{\mathrm{f}}(-\mathbf{k})\right\rangle} \\
S_{\mathrm{c}}(k)=\frac{1}{N_{\mathrm{f}}} \overline{\left\langle\delta \rho_{\mathrm{f}}(\mathbf{k}) \delta \rho_{\mathrm{f}}(-\mathbf{k})\right\rangle},
\end{gathered}
$$

where $\delta \rho_{i}(\mathbf{k})=\rho_{i}(\mathbf{k})-\left\langle\rho_{i}(\mathbf{k})\right\rangle$ with $i=\mathrm{m}$ or $\mathrm{f}$. In these expressions $\langle\cdots\rangle$ denotes the thermal average over the degrees of freedom of the fluid particles at a given matrix configuration, while $\cdots$ stands for the average over different, but equivalent matrix configurations. The wave vectors $\mathbf{k}$ are chosen to be compatible with the cubic simulation box, i.e., $\mathbf{k}=(2 \pi / L)\left(n_{x}, n_{y}, n_{z}\right)$, with the $n_{i}$ being integers. We point out that a straightforward evaluation of $S_{\mathrm{b}}(k)$ does not necessarily guarantee for a proper decay of this structure factor for large $k$-values. Numerical problems related to the evaluation of $S_{\mathrm{b}}(k)$ will be discussed in Section 3.2.

\section{Results}

In this section we describe the structural properties of the quenched-annealed GCM system. We report results of MC simulations and integral-equation theory for densities $\rho_{\mathrm{f}}$ and $\rho_{\mathrm{m}}$ in the range $0.05 \leq \rho_{\mathrm{f}} \leq 0.50$ and $0.05 \leq \rho_{\mathrm{m}} \leq 0.30$. In most simulations, we kept the temperature of the matrix, $T_{\mathrm{m}}$, and that of the fluid, $T_{\mathrm{f}}$, equal to each other and fixed at 0.01, i.e., slightly above the upper freezing temperature of the equilibrium GCM fluid [31]. In a few cases, we also varied $T_{\mathrm{m}}$ and $T_{\mathrm{f}}$ independently to investigate the effect of a temperature mismatch between the fluid and the matrix.

\subsection{Fluid-fluid correlations}

To characterise the structure of our model we used the structure factors introduced in Section 2. We start our discussion from the fluid-fluid structure factor $S_{\mathrm{ff}}(k)$. In the left panel of Figure 1 we show results of MC simulations for different fluid densities at constant matrix density $\rho_{\mathrm{m}}=0.1$, along with the corresponding solutions of the ROZ equations [Equations (2)-(5)] complemented by the HNC approximation [Equation (6)]. The right panel of Figure 1 displays the corresponding data obtained at higher matrix density $\left(\rho_{\mathrm{m}}=0.3\right)$. Comparison between theoretical 

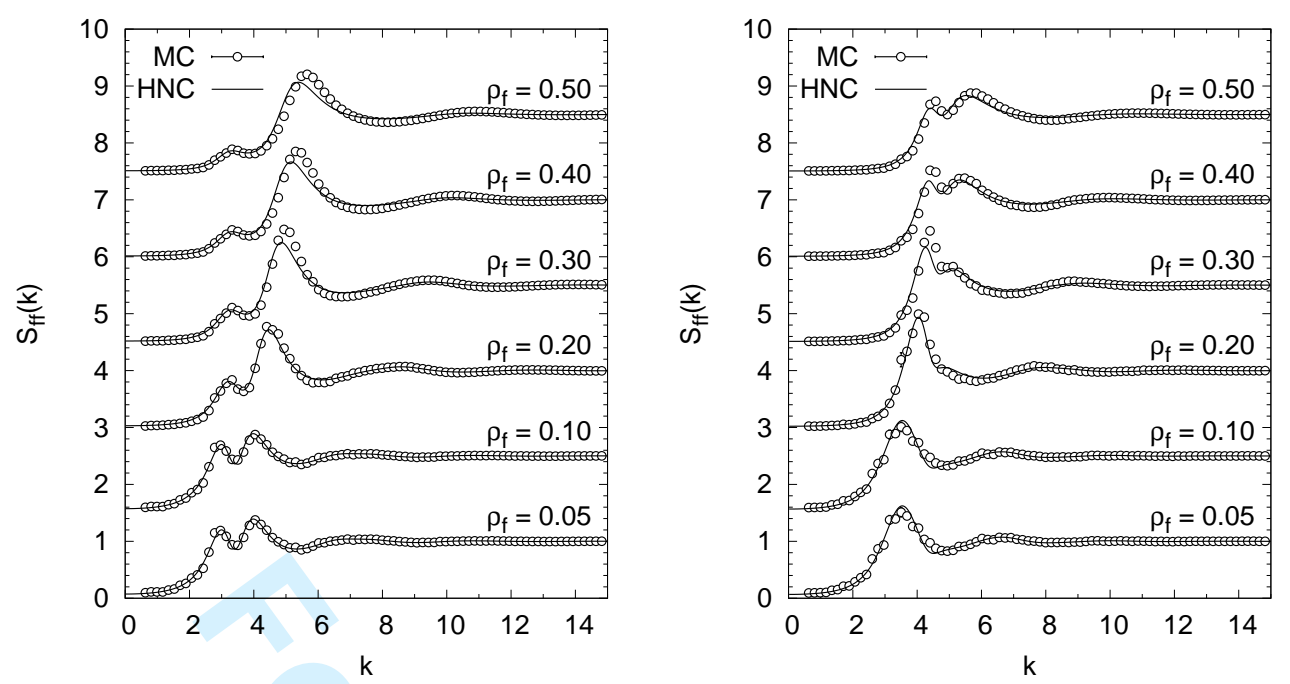

Figure 1. Fluid-fluid structure factors $S_{\mathrm{ff}}(k)$ as a function of $k$ at $\rho_{\mathrm{m}}=0.1$ (left panel) and $\rho_{\mathrm{m}}=0.3$ (right panel) for different values of $\rho_{\mathrm{f}}$. The temperatures are $T_{\mathrm{f}}=T_{\mathrm{m}}=0.01$. Symbols and solid lines indicate results from MC simulations and solutions of the ROZ equations (HNC approximation), respectively. Different data sets have been displaced in vertical direction by 1.5 .

predictions and simulation results shows that the HNC closure is able to account for fluid-fluid correlations at a quantitative level in the whole investigated density regime. In the light of previous studies on both equilibrium and quenched-annealed systems [5, 31, 39], the effectiveness of the HNC approximation in describing fluidfluid correlations of the present model is unsurprising. On the one hand, in fact, it is known that HNC provides a satisfactory description of the structure of QA systems of hard spheres $[5,39]$. On the other hand, HNC is also regarded as a reliable closure for the equilibrium GCM fluid [31] and, more generally, for fluids with soft interactions [20].

A remarkable feature is also apparent in Figure 1. For $\rho_{\mathrm{m}}=0.1$ and all studied fluid densities (see left panel), $S_{\mathrm{ff}}(k)$ displays a distinct pre-peak located at small wave-vectors $(k \sim 3)$. Anticipating the discussion given below, this pre-peak in the fluid-fluid structure factor is a manifestation of the underlying structure of the matrix. For $\rho_{\mathrm{f}} \gtrsim \rho_{\mathrm{m}}$ the height of the pre-peak decreases with increasing fluid density, while its position remains essentially constant. In the same density regime, the main peak displays a more complex evolution, which resembles the behaviour of the equilibrium GCM fluid [31]. In fact, the position of the main peak shifts markedly to larger $k$ with increasing density, while its height displays a non-monotonic behaviour. Finally, we note that a similar "double-peak" shape is present also at $\rho_{\mathrm{m}}=0.3$ and high fluid densities (see right panel of Figure 1). In this latter case, however, the pre-peak is shifted to $k \sim 4$ and eventually merges 
Figure 2. Height of the main peak of $S_{\mathrm{ff}}(k)$ determined from MC simulations as a function of $\rho_{\mathrm{f}}$ for different values of $\rho_{\mathrm{m}}\left(T_{\mathrm{f}}=T_{\mathrm{m}}=0.01\right)$.

with the main peak at low $\rho_{\mathrm{f}}$.

The non-monotonic behaviour of the main peak in $S_{\mathrm{ff}}(k)$ is evidenced more clearly in Figure 2, where the height of the main peak is plotted as a function of $\rho_{\mathrm{f}}$ for selected values of $\rho_{\mathrm{m}}$. In particular, the height always attains a maximum in the considered range of fluid density. Lang et al. [31] found indeed qualitatively similar features in the variation of the structure factor of the equilibrium GCM fluid. They also showed that the non-monotonic behaviour of the peak height is strictly related to the re-entrant melting transition of the equilibrium GCM [31]. In the QA system (see Figure 2) the increase of the matrix density tends to suppress fluidfluid correlations. This points to a key role of the matrix structure in frustrating the crystallisation of the adsorbed fluid.

To gain a better understanding of the structural features apparent in Figure 1, it is useful to compare the QA model with two reference equilibrium fluids at different densities: The first at a density $\rho=\rho_{\mathrm{m}}$, corresponding to the equilibrium fluid from which the matrix configurations were quenched; The second at a density equal to the total density of the QA system, i.e, $\rho=\rho_{\mathrm{f}}+\rho_{\mathrm{m}}$. Both reference fluids are considered at the same temperature $T=T_{\mathrm{m}}=T_{\mathrm{f}}$. This procedure is similar to the "generalised percolation approximation" used in Ref. [5] to describe the total connected correlation function of QA hard-sphere models at moderate and high porosity. $^{1}$

\footnotetext{
${ }^{1}$ In the generalised percolation approximation, however, the density of the reference equilibrium fluid is $\rho=\rho_{\mathrm{f}} / H\left(\rho_{\mathrm{m}}\right)$, where $H\left(\rho_{\mathrm{m}}\right)$ is the Henry constant [5]. For a QA hard-sphere system, $H$ measures the fraction of volume available to the centre of a fluid particle adsorbed in a matrix of density $\rho_{\mathrm{m}}$.
} 


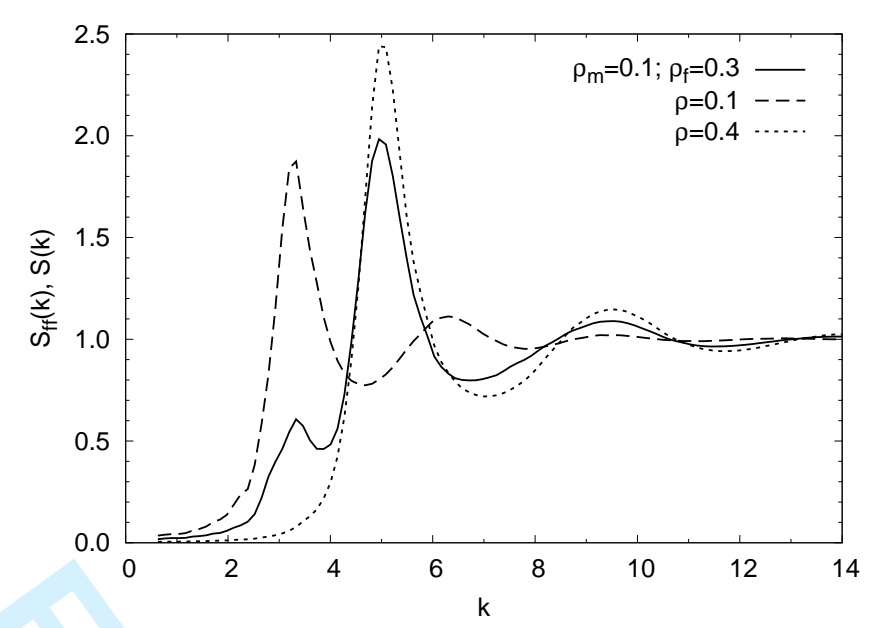

Figure 3. Fluid-fluid structure factor $S_{\mathrm{ff}}(k)$ determined from MC simulations as a function of $k$ at $\rho_{\mathrm{m}}=$ 0.1 and $\rho_{\mathrm{f}}=0.3$ (solid line). Also shown are the structure factors $S(k)$ of equilibrium fluids at $\rho=\rho_{\mathrm{m}}$ (dotted line) and at $\rho=\rho_{\mathrm{m}}+\rho_{\mathrm{f}}$ (dashed line).

In Figure 3 we compare the fluid-fluid correlations of the QA system to those of the reference fluids for one selected state point $\left(\rho_{\mathrm{m}}=0.1, \rho_{\mathrm{f}}=0.3, T_{\mathrm{m}}=\right.$ $\left.T_{\mathrm{f}}=0.01\right)$. This state point is representative of the behaviour of the model at intermediate matrix density and intermediate and high fluid densities $\left(\rho_{\mathrm{f}} \gtrsim \rho_{\mathrm{m}}\right)$. The comparison reveals that the pre-peak of $S_{\mathrm{ff}}(k)$ appears exactly at the same wave-vector as the main peak of $S(k)$ of the equilibrium fluid at $\rho=\rho_{\mathrm{m}}$. Hence, this feature of the QA system originates in the properties of the equilibrium fluid from which the matrix configurations were quenched. On the other hand, the position of the main peak of $S_{\mathrm{ff}}(k)$ corresponds to the peak of $S(k)$ of the equilibrium fluid at density $\rho=\rho_{\mathrm{f}}+\rho_{\mathrm{m}}$. Indeed we found that the fluid-fluid radial distribution function $g_{\mathrm{ff}}(r)=h_{\mathrm{ff}}(r)+1$ (not displayed here) oscillates in phase with the $g(r)$ of the equilibrium fluid at $\rho=\rho_{\mathrm{m}}+\rho_{\mathrm{f}}$. Thus, it seems that the essential features of $S_{\mathrm{ff}}(k)$ can be reasonably explained in terms of the typical emerging order of equilibrium fluids, at least for not too low matrix density and $\rho_{\mathrm{f}} \gtrsim \rho_{\mathrm{m}}$. At fluid densities comparable to $\rho_{\mathrm{m}}$ this interpretation is no longer valid. In this latter case, however, the distinction between pre-peak and main peak also becomes less clear (see Figure 1). Finally, we note that, in the more general case in which fluid-fluid, fluid-matrix, and matrix-matrix interactions differ, one should rather consider an equilibrium mixture at density $\rho=\rho_{\mathrm{m}}+\rho_{\mathrm{f}}$ as a reference fluid for the QA system, with the two components having the appropriate chemical concentrations. 

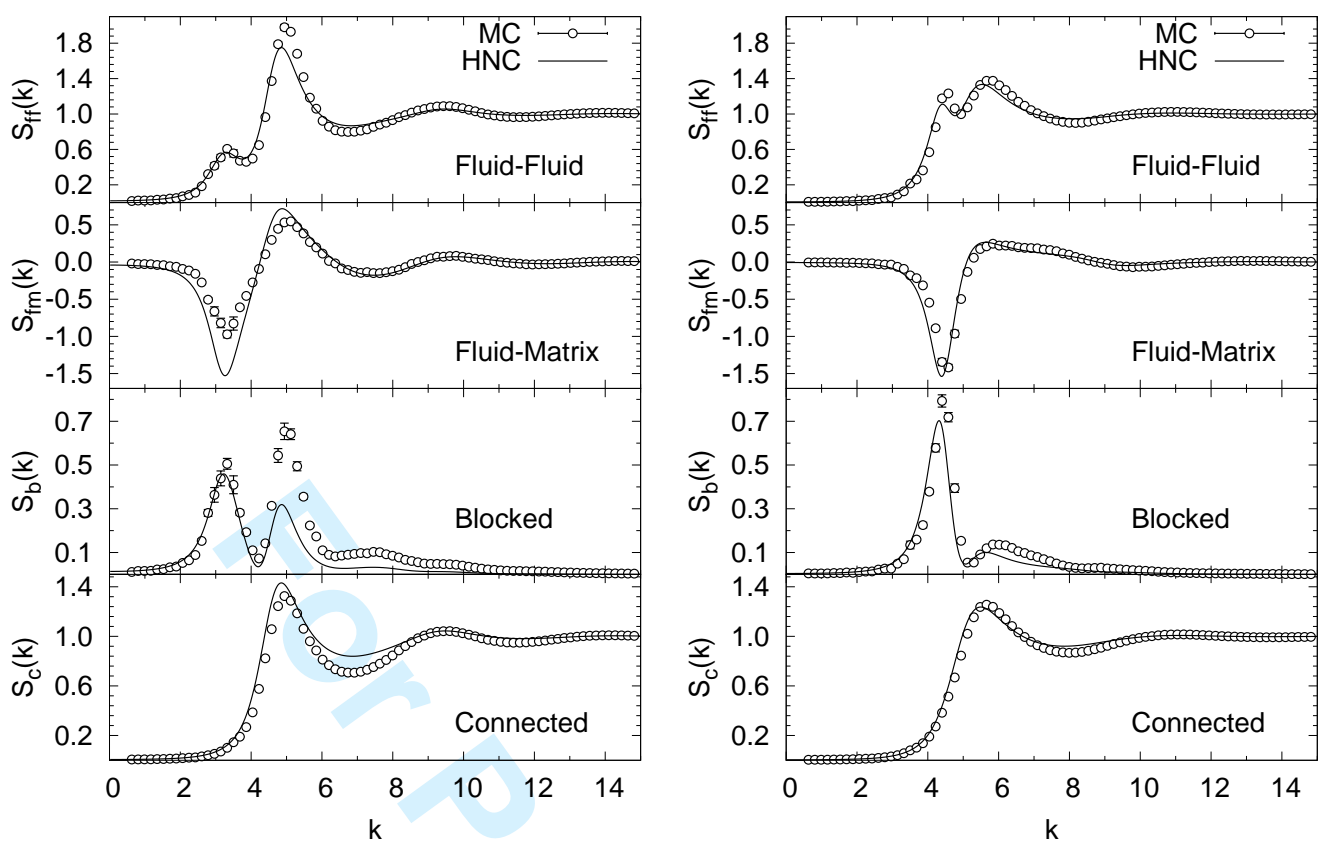

Figure 4. Comparison of MC (symbols) and HNC (solid lines) results for $S_{\mathrm{ff}}(k), S_{\mathrm{fm}}(k), S_{\mathrm{c}}(k)$, and $S_{\mathrm{b}}(k)$ as a function of $k$. The state points considered are $\rho_{\mathrm{m}}=0.1, \rho_{\mathrm{f}}=0.3$ (left panel) and $\rho_{\mathrm{m}}=0.3, \rho_{\mathrm{f}}=0.5$ (right panel), both at $T_{\mathrm{m}}=T_{\mathrm{f}}=0.01$.

\subsection{Role of blocked correlations}

An even sharper interpretation of the structural features discussed above is achieved by splitting $S_{\mathrm{ff}}(k)$ into its blocked and connected parts according to Equations (15) and (16) [remember that $\left.S_{\mathrm{ff}}(k)=S_{\mathrm{b}}(k)+S_{\mathrm{c}}(k)\right]$. In Figure 4 we plot $S_{\mathrm{b}}(k), S_{\mathrm{c}}(k)$, $S_{\mathrm{ff}}(k)$, and $S_{\mathrm{fm}}(k)$ for two representative state points: $\rho_{\mathrm{m}}=0.1, \rho_{\mathrm{f}}=0.3$ (left panel) and $\rho_{\mathrm{m}}=0.3, \rho_{\mathrm{f}}=0.5$ (right panel). A closer look at the data reveals that in both cases the pre-peak can be fully ascribed to blocked correlations. In fact, the position of the pre-peak in $S_{\mathrm{ff}}(k)$ coincides exactly with the first peak of $S_{\mathrm{b}}(k)$. Moreover, the "double-peak" structure is absent in the connected part $S_{\mathrm{c}}(k)$. Since the blocked structure factor accounts for correlations between fluid particles mediated by at least one matrix particle [5], the results in Figure 4 confirm that the pre-peak in $S_{\mathrm{ff}}(k)$ is an effect induced by the matrix structure on the fluid-fluid correlations.

Figure 4 also shows that the solutions of the ROZ equations in combination with the HNC closure overall are in good agreement with the simulation data, but some discrepancies appear for $S_{\mathrm{b}}(k)$ and $S_{\mathrm{fm}}(k)$ (see left panel). In particular, the relative discrepancy between the data sets in the case of blocked correlations 


\section{Molecular Physics}

becomes more pronounced for $k$ values around and beyond the main peak position in $S_{\mathrm{ff}}(k)$. In this case, the disagreement might not be charged entirely to the theory. In fact, Meroni and coworkers [39] showed that real-space blocked correlations determined from numerical simulations may be affected by a slowly decaying "selfterm", which makes $g_{\mathrm{b}}(r)$ inaccurate for $r \approx 0$. We found that similar difficulties are present in Fourier space in the asymptotic limit of large wave-vectors. ${ }^{1}$ To overcome these problems we used the simple trick suggested in Ref. [39] for the $r$ space representation of the correlation functions. The averages $\left\langle\rho_{\mathrm{f}}(\mathbf{k})\right\rangle$ and $\left\langle\rho_{\mathrm{f}}(-\mathbf{k})\right\rangle$ were evaluated during different parts of the simulation: while $\left\langle\rho_{\mathrm{f}}(\mathbf{k})\right\rangle$ was calculated during the first half, $\left\langle\rho_{\mathrm{f}}(-\mathbf{k})\right\rangle$ was evaluated during the second half of the run. Nonetheless, a fully accurate determination of blocked correlations from simulations might still be hindered by finite-size effects, which we have not analysed yet in a systematic way. Further work is probably needed to clarify this issue.

The observations we made in discussing Figure 3 and 4 agree with the consensus on the strong influence of the matrix on the properties of the adsorbed fluid [3, 40]. We note, however, that in our model the effect of the matrix on fluid-fluid correlations, which appears at low wave-vectors, is essentially "geometric" in nature. We will argue below that the pre-peak in $S_{\mathrm{ff}}(k)$ is a more general feature of QA models with purely repulsive fluid-matrix interactions. On the other hand, Krakoviack et al. [40] observed a similar - yet much more pronounced - correlation peak in $S_{\mathrm{ff}}(k)$ at low wave-vectors in a model of fluid adsorbed in highly porous aerogel. They attributed such correlation peaks to the presence of attractive fluid-matrix interactions, which caused fluid particles to coat the aerogel strands. Interestingly, the different origin of the pre-peak in these models may be related to the shape of $S_{\mathrm{fm}}(k)$. In our purely repulsive model, the pre-peak in $S_{\mathrm{ff}}(k)$ always occurs at wave-vectors at which fluid-matrix correlations are suppressed. In contrast, in the model aerogel of Ref. [40] fluid-matrix correlations were generally enhanced at low $k$.

\footnotetext{
${ }^{1}$ The presence of a slowly decaying "self-term" in the blocked structure factors determined from simulations can be seen by inserting the expression of the one-particle density $\rho_{\mathrm{f}}(k)$ [see Equation (12)] into Equation (15). Contributions to the product in Equation (15) coming from equal particles at equal time steps give rise to a term equal to $1 / M$ in $S_{\mathrm{b}}(k)$, where $M$ is the number of configurations used for averaging. Moreover, $\left\langle\rho_{\mathrm{f}}(k)\right\rangle$ determined from simulations is affected by statistical uncertainty, which shows up as a positive background noise in $S_{\mathrm{b}}(k)$. These features prevent $S_{\mathrm{b}}(k)$ [as defined in Equation (15)] from decaying fully to zero at very large $k$.
} 


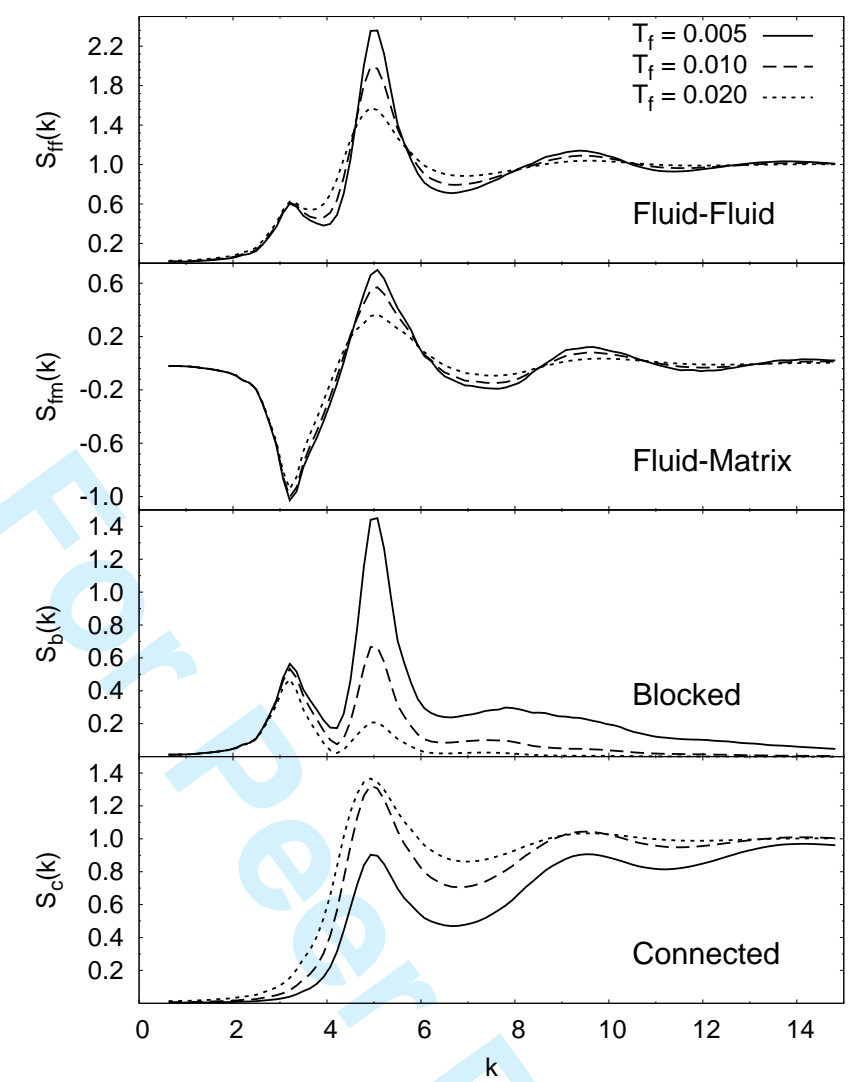

Figure 5. Fluid-fluid $S_{\mathrm{ff}}(k)$, fluid-matrix $S_{\mathrm{fm}}(k)$, blocked $S_{\mathrm{b}}(k)$, and connected $S_{\mathrm{c}}(k)$ structure factors as function of $k$. Results refer to MC simulations at different fluid temperatures $T_{\mathrm{f}}$ and at fixed $T_{\mathrm{m}}=0.01$. The density of the matrix and the fluid are $\rho_{\mathrm{m}}=0.1$ and $\rho_{\mathrm{f}}=0.3$, respectively.

\subsection{Temperature mismatch}

So far, we considered only systems in which the temperature of the fluid, $T_{\mathrm{f}}$, and the temperature of the matrix, $T_{\mathrm{m}}$, were equal. ${ }^{1}$ It is interesting to investigate the respective roles of these parameters. The possibility of a temperature mismatch has been noted in previous work [41], but an explicit assessment of its consequences has not been carried out yet.

Let us first investigate the role of $T_{\mathrm{f}}$ at fixed $T_{\mathrm{m}}$. The temperature evolution of $S_{\mathrm{ff}}(k)$ obtained from MC simulations is shown in Figure 5 for the representative state point $\rho_{\mathrm{m}}=0.1, \rho_{\mathrm{f}}=0.3$ (i.e., densities as in the left panel of Figure 4). The increase in height of the main peak upon lowering $T_{\mathrm{f}}$ indicates a progressive ordering of the fluid inside the matrix. In contrast, the pre-peak does not show

\footnotetext{
${ }^{1}$ Remember that $T_{\mathrm{m}}$ is the temperature of the equilibrium fluid from which we obtained the matrix configurations, as described in Section 2.
} 


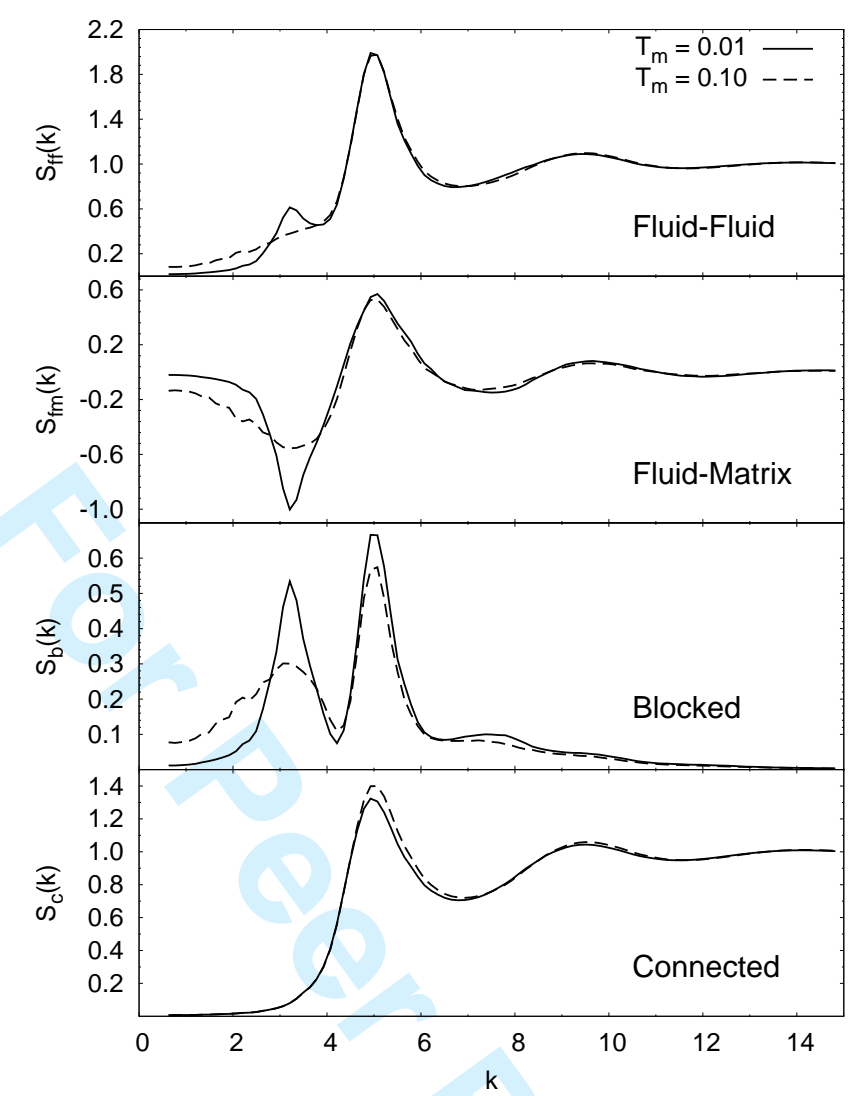

Figure 6. Fluid-fluid $S_{\mathrm{ff}}(k)$, fluid-matrix $S_{\mathrm{fm}}(k)$, blocked $S_{\mathrm{b}}(k)$, and connected $S_{\mathrm{c}}(k)$ structure factors as function of $k$. Results refer to MC simulations at different matrix temperatures $T_{\mathrm{m}}$ and at fixed $T_{\mathrm{f}}=0.01$. The density of the matrix and the fluid are $\rho_{\mathrm{m}}=0.1$ and $\rho_{\mathrm{f}}=0.3$, respectively.

any substantial variation upon cooling. Hence, the structural features observed at low wave-vectors in our model cannot be simply understood in terms of the formation of some intermediate-range order - similar to the one observed upon cooling in network-forming liquids [42]. In the latter case, in fact, the peak at low wave-vectors associated to intermediate-range order grows by decreasing $T$ while the height of the main peak decreases.

It is perhaps even more revealing to study the variation of the fluid structure when $T_{\mathrm{m}}$ is changed at fixed $T_{\mathrm{f}}$. In this way we can investigate in a controlled manner the role of correlations in the matrix structure. The structure factors $S_{\mathrm{ff}}(k)$, $S_{\mathrm{fm}}(k), S_{\mathrm{c}}(k)$, and $S_{\mathrm{b}}(k)$ are shown in Figure 6 for the same selected state point of Figure 4 and 5 , but for two different values of $T_{\mathrm{m}}$. Matrix configurations sampled at high temperature $\left(T_{\mathrm{m}}=0.1\right)$ alter the shape of the structure factors $S_{\mathrm{ff}}(k), S_{\mathrm{fm}}(k)$, and $S_{\mathrm{b}}(k)$, leading to a substantial rounding of the features at low wave-vectors in 


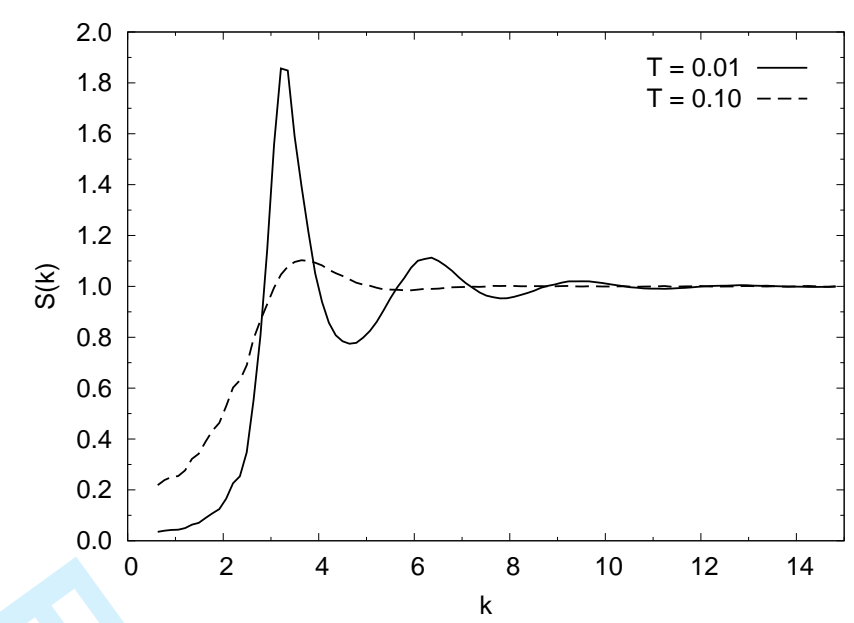

Figure 7. Structure factors of the equilibrium GCM fluid obtained from MC simulations at $\rho=0.1$ for $T=0.1$ (dashed line) and $T=0.01$ (solid line).

$S_{\mathrm{ff}}(k)$. Upon lowering $T_{\mathrm{m}}$, correlations in the matrix structure are enhanced (see the $S(k)$ of the corresponding equilibrium fluids in Figure 7) and they affect more strongly the structure of the fluid at low wave-vectors. On the other hand, the connected part $S_{\mathrm{c}}(k)$, which describes fluid correlations not mediated by the matrix, is essentially unaltered by changes in the matrix structure due to the different matrix temperature. Hence, temperature mismatch between fluid and matrix represents a simple tool to probe the sensitivity of fluid correlations to the underlying matrix structure and to pin down the relevant wave-vectors at which matrix-induced effects are more pronounced.

\section{Discussion and conclusions}

In this work we have analysed, using integral-equation theory and Monte Carlo simulations, the structural properties of a model colloidal fluid imbibed in porous confinement. The porous matrix consisted of quenched configurations of the same colloidal fluid, i.e., particles with the same nominal diameter. While the matrix structure we employed is unrealistic compared to typical porous materials considered in the literature (e.g., aerogels, vycor, controlled glasses), it might be experimentally realised by pinning particles at well-defined positions using optical tweezers on colloidal suspensions [19, 43, 44]. Hence, the choice of a model that describes the effective interactions between linear polymer chains represents a step ahead towards closer contact between theory and experiment. Arguably, novel features may also appear when soft matter is adsorbed in porous media. 


\section{Molecular Physics}

16

D. F. Schwanzer et al.

Some of the variations observed in the fluid structure factors when changing the fluid density at constant matrix density resembled the behaviour of the equilibrium GCM fluid. Specifically, the height of the main peak of $S_{\mathrm{ff}}(k)$ changed in a nonmonotonic fashion - a behaviour peculiar of soft matter for re-entrant solidification scenario. On the other hand, we found a distinct correlation peak in $S_{\mathrm{ff}}(k)$ at wave-vectors corresponding to the the main peak of the matrix-matrix structure factor. Such a pre-peak was especially apparent at intermediate fluid densities and was interpreted as an indirect effect of the matrix geometry. We argue now that this feature is of more general nature and not specific of our model. First, from preliminary, unpublished results we found a similar pre-peak structure in the same model but with different values of size ratio $\lambda=\sigma_{\mathrm{ff}} / \sigma_{\mathrm{fm}}$ (namely $\lambda=1.4$ and $\lambda=3.0)$. Second, a mismatch between the peak position of blocked and connected structure factors in a QA system of hard spheres has been recently reported by Krakoviack [38]. Indeed, our simulations for the QA hard-sphere model confirmed the presence of a pre-peak in $S_{\mathrm{ff}}(k)$ for intermediate porosity. Finally, a correlation peak in $S_{\mathrm{ff}}(k)$ has been observed at intermediate fluid densities in a model of fluid adsorbed in a highly porous aerogel [40]. The latter model featured a fractal matrix structure and attractive fluid-matrix interactions, utterly different from our model.

Our analysis has also highlighted the usefulness of blocked correlations in explaining distinct features of structural properties of fluids adsorbed in porous media. The work by Jean-Jacques Weis and coworkers $[5,8-12,39,45,46]$ has provided a deeper understanding of the properties of fluids in porous media in general and of blocked correlation functions in particular, both from a theoretical and computational point of view. Our work indicates that more effort will be required to accurately compute these correlations functions. This is crucial in the light of the key role played by blocked and connected correlations in a novel formulation of Mode Coupling theory for describing the dynamics of fluids in porous materials [38].

\section{Acknowledgements}

Financial support by the Austrian Research Fund (FWF) under Proj. Nos. P17823N08, P19890-N16 and W004 is gratefully acknowledged. 


\section{References}

[1]M. Chan, N. Mulders and J. Reppy, Phys. Today 49, 30 (1996).

[2] M.L. Rosinberg, in New Approaches to problems in liquid state theory, edited by C. Caccamo, J.P. Hansen and G. Stell (Kluwer Academic, Dordrecht, AA, The Netherlands, 1999).

[3]L.D. Gelb, K.E. Gubbins, R. Radhakrishnan and M. Sliwinska-Bartkowiak, Rep. Prog. Phys. 62, 1573 (1999).

[4]E. Kierlik, M.L. Rosinberg, G. Tarjus and P.A. Monson, J. Chem. Phys. 106, 264 (1997).

[5]E. Lomba, J.A. Given, G. Stell et al., Phys. Rev. E 48, 233 (1993).

[6] M.J. Fernaud, E. Lomba and L.L. Lee, J. Chem. Phys 111, 10275 (1999).

[7]E. Paschinger and G. Kahl, Phys. Rev. E 61, 5330 (2000).

[8]E. Paschinger, D. Levesque, G. Kahl and J.J. Weis, Europhys. Let. 55 (2), 178 (2001).

[9]E. Schöll-Paschinger, D. Levesque, J.J. Weis and G. Kahl, Phys. Rev. E 64 (1), 011502 (2001).

[10] M.J. Fernaud, E. Lomba and J.J. Weis, Phys. Rev. E 64, 051501 (2001).

[11] M.J. Fernaud, E. Lomba, J.J. Weis and D. Levesque, Mol. Phys. 101, 1721 (2003).

[12] M.J. Fernaud, E. Lomba, C. Martín et al., J. Chem. Phys. 119, 364 (2003).

[13]C. Spoler and S.H.L. Klapp, J. Chem. Phys. 118, 3628 (2003).

[14]M.J. Fernaud and E. Lomba, in Ionic Soft Matter: Modern trends in theory and applications and NATO Science Series: II - Mathematics and Physics and Chemistry, vol. 206., edited by M. Holovko D. Henderson and A. Trokhymchuk (Kluwer Springer, Dordrecht, AA, The Netherlands, 2005).

[15]M.J. Fernaud, G. Kahl and C.N. Likos, Eur. Phys. J. Special Topics 141, 251 (2007).

[16]R.L.C. Vink, K. Binder and H. Löwen, Phys. Rev. Let. 97, 230603 (2006).

[17] J.C. Crocker and D.G. Grier, J. Colloid Interface Sci. 179, 298 (1996).

[18]A. van Blaaderen and P. Wiltzins, Science 270, 1177 (1995).

[19]D.G. Grier, Nature 424, 810 (2003).

[20] C.N. Likos, Phys. Rep. 348, 267 (2001).

[21]W. Dong, V. Krakoviack and S.L. Zhao, J. Phys. Chem. C 111 (43), 15910 (2007).

[22] S.L. Zhao, W. Dong and Q.H. Liu, J. Chem. Phys. 125 (24), 244703 (2006).

[23]L. Sarkisov and P.R. Van Tassel, J. Phys.: Condens. Matter 20, 333101 (2008).

[24]P.R. Van Tassel, Phys. Rev. E 60, R25 (1999).

[25]L. Zhang, S. Cheng and P.R. Van Tassel, Phys. Rev. E 64, 042101 (2001).

[26] L. Zhang and P.R. Van Tassel, J. Chem. Phys. 112, 3006 (2000).

[27]P.J. Flory and W.R. Krigbaum, J. Chem. Phys. 18, 1086 (1950).

[28]F.H. Stillinger, J. Chem. Phys. 65, 3968 (1976).

[29] W.G. Madden and E. Glandt, J. Stat. Phys. 51, 537 (1988).

[30] J.A. Given and G. Stell, J. Chem. Phys. 97, 4573 (1992).

[31] A. Lang, C.N. Likos, M. Watzlawek and H. Löwen, J. Phys.: Condens. Matter 12, 5087 (2000).

[32]S. Prestipino, F. Saija and P.V. Giaquinta, Phys. Rev. E 71 (5), 050102 (2005).

[33] S.F. Edwards and P.W. Anderson, J. Phys. F: Metal Phys. 5, 965 (1975).

[34] J.P. Hansen and I.R. McDonald, Theory of Simple Liquids, 3rd ed. (Academic Press, London, 2006).

[35] C.N. Likos, J. Chem. Phys. 126, 224502 (1997).

[36] A.Z. Panagiotopoulos, J. Chem. Phys. 112, 7132 (2000).

[37]D. Frenkel and B. Smit, Understanding Molecular Simulation, 2nd ed. (Academic Press, London, 2002).

[38]V. Krakoviack, Phys. Rev. E 75, 031503 (2007).

[39] A. Meroni, D. Levesque and J.J. Weis, J. Chem. Phys. 105, 1101 (1996).

[40]V. Krakoviack, E. Kierlik, M.L. Rosinberg and G. Tarjus, J. Chem. Phys. 115, 11289 (2001).

[41] M.L. Rosinberg, G. Tarjus and G. Stell, J. Chem. Phys. 100, 5172 (1994).

[42] J. Horbach and W. Kob, Phys. Rev. B 60, 3169 (1999).

[43] P.J. Rodrigo, V.R. Daria and J. Gluckstad, Appl. Phys. Lett. 86, 074103 (2005).

[44]D.L.J. Vossen, A. van der Horst, M. Dogterom and A. van Blaaderen, Rev. Sci. Instrum. 75, 2960 
(2004).

[45]M. Álvarez, D. Levesque and J.J. Weis, Phys. Rev. E 60 (5), 5495-5504 (1999).

[46]E. Schöll-Paschinger, D. Levesque, J.J. Weis and G. Kahl, J. Chem. Phys. 122, 24507-1 (2005). 


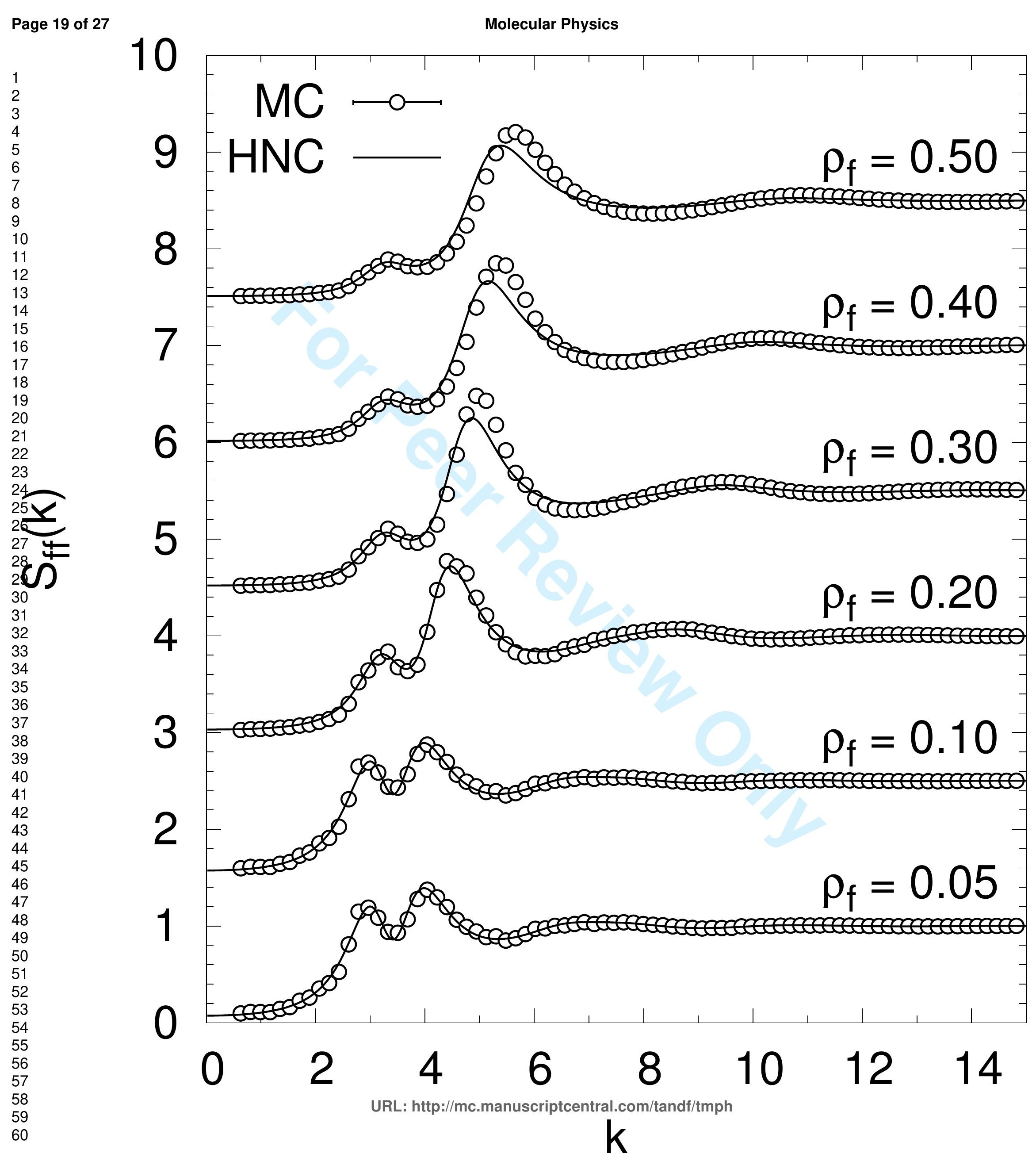




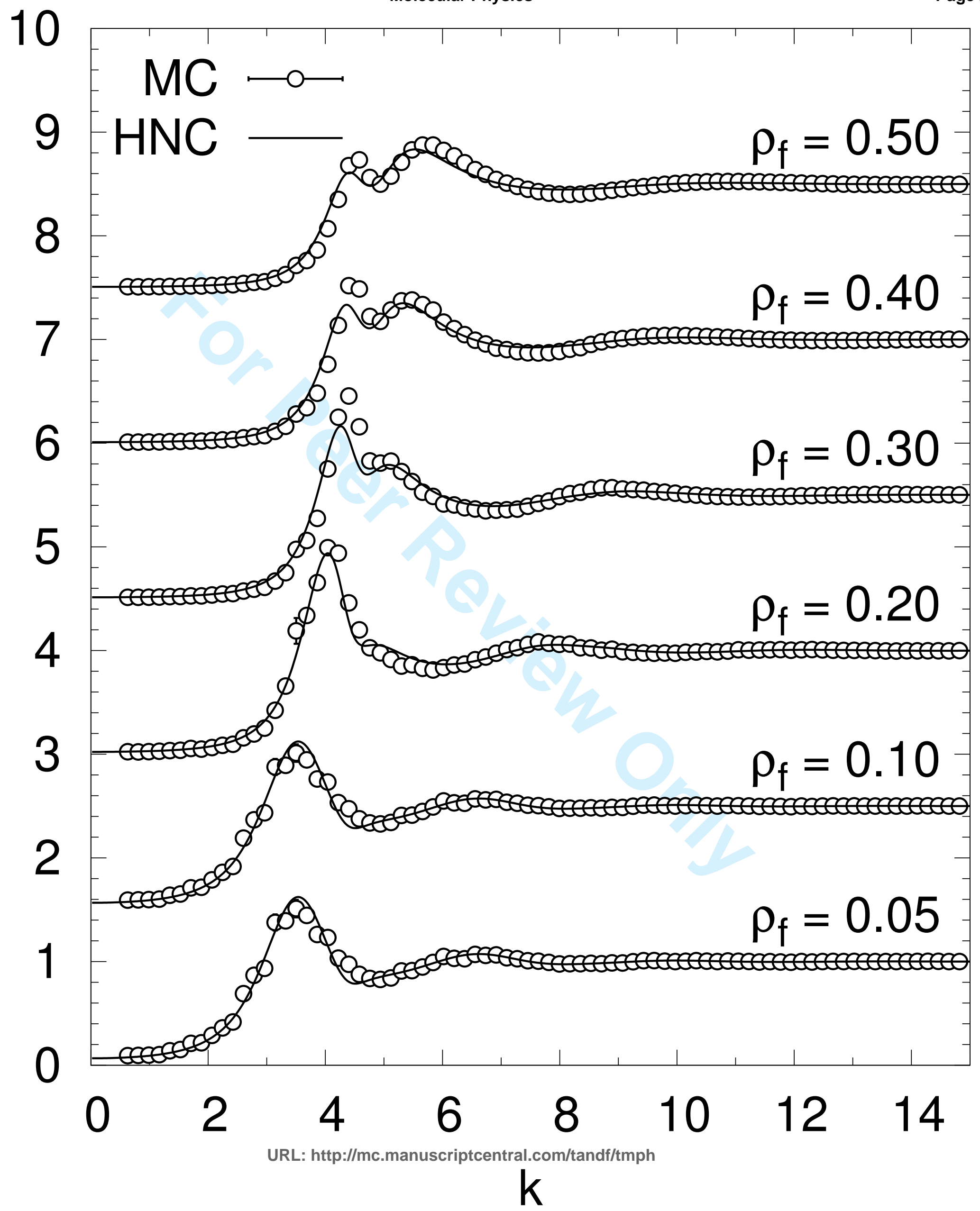




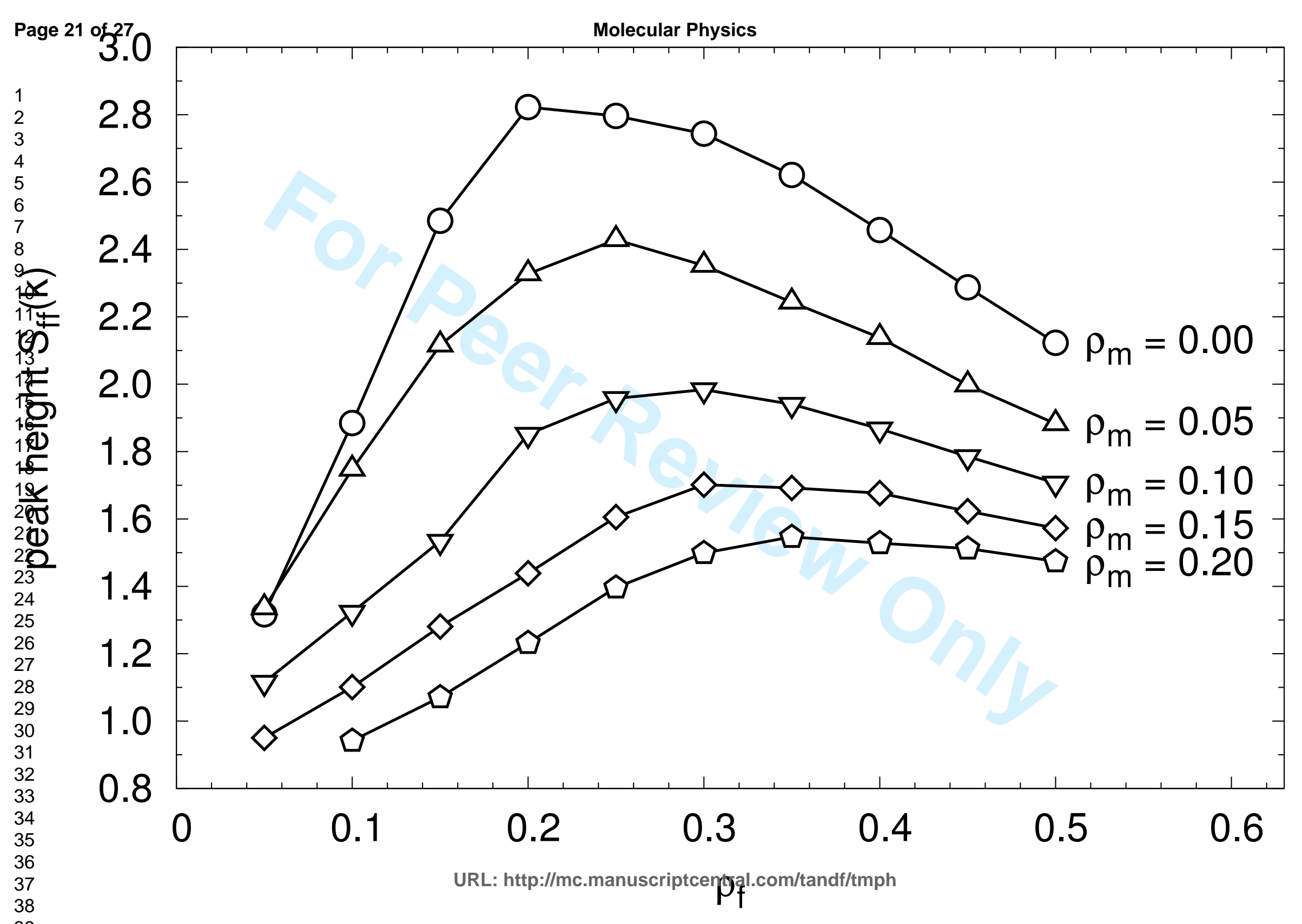




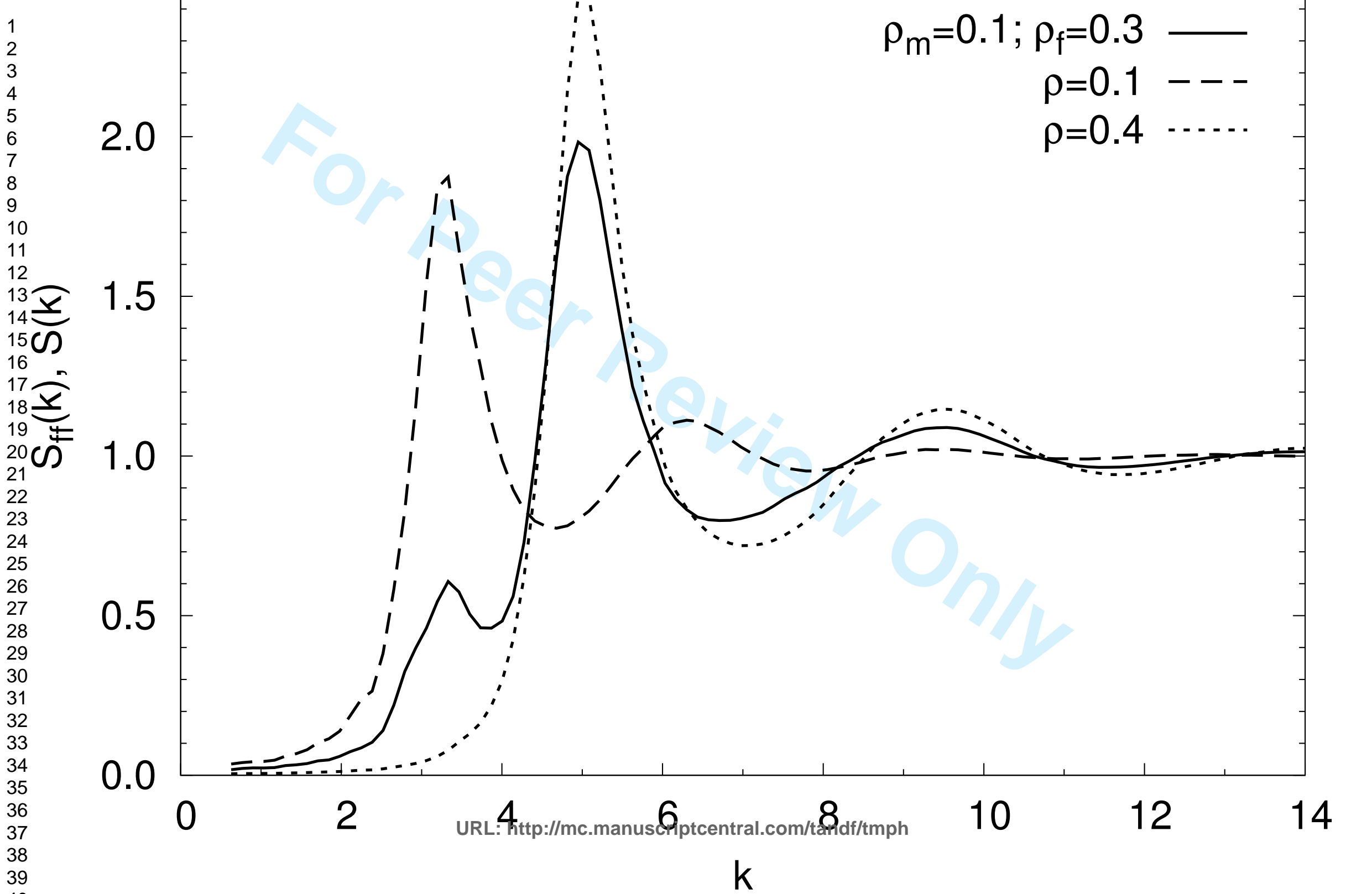




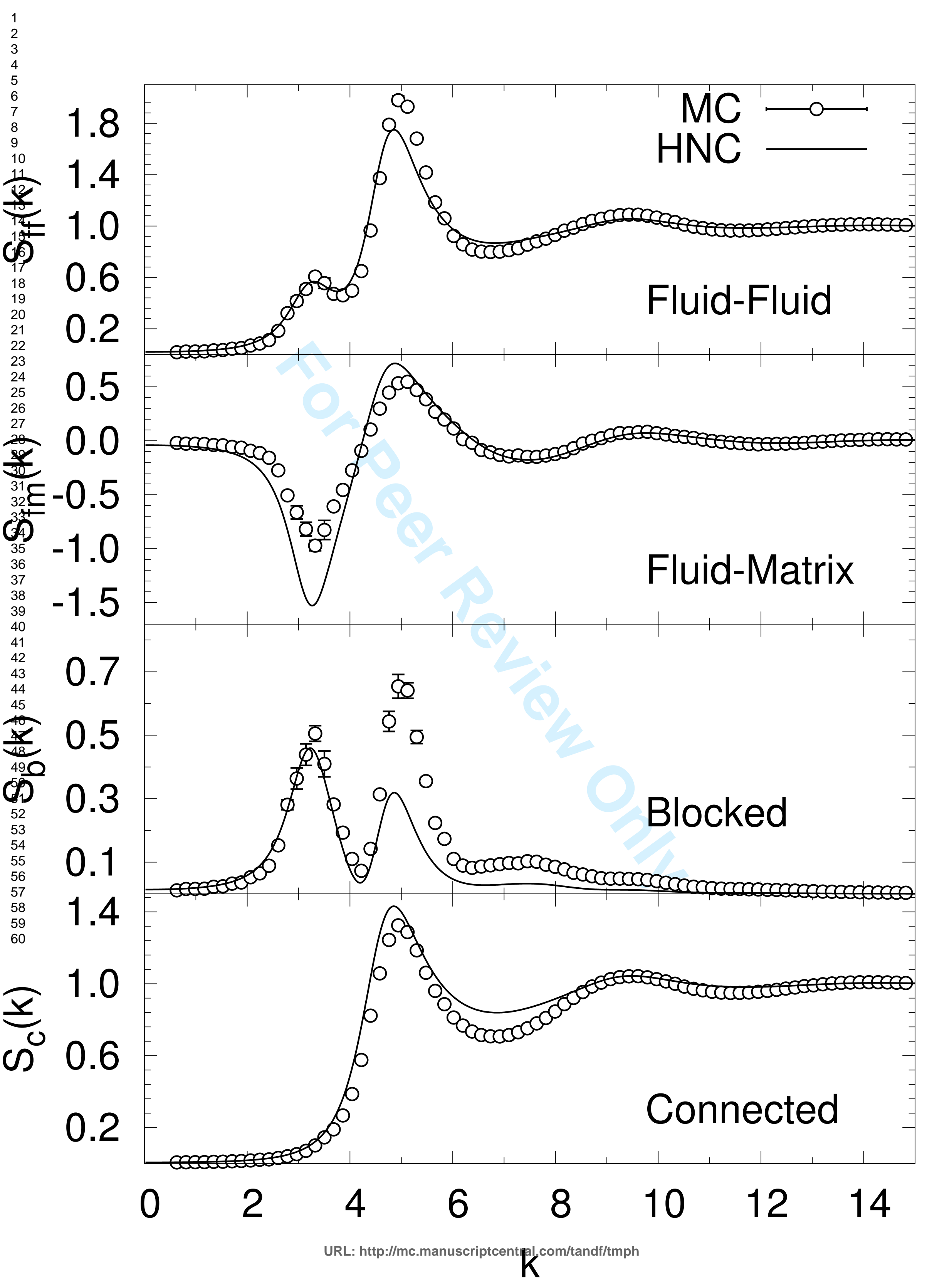




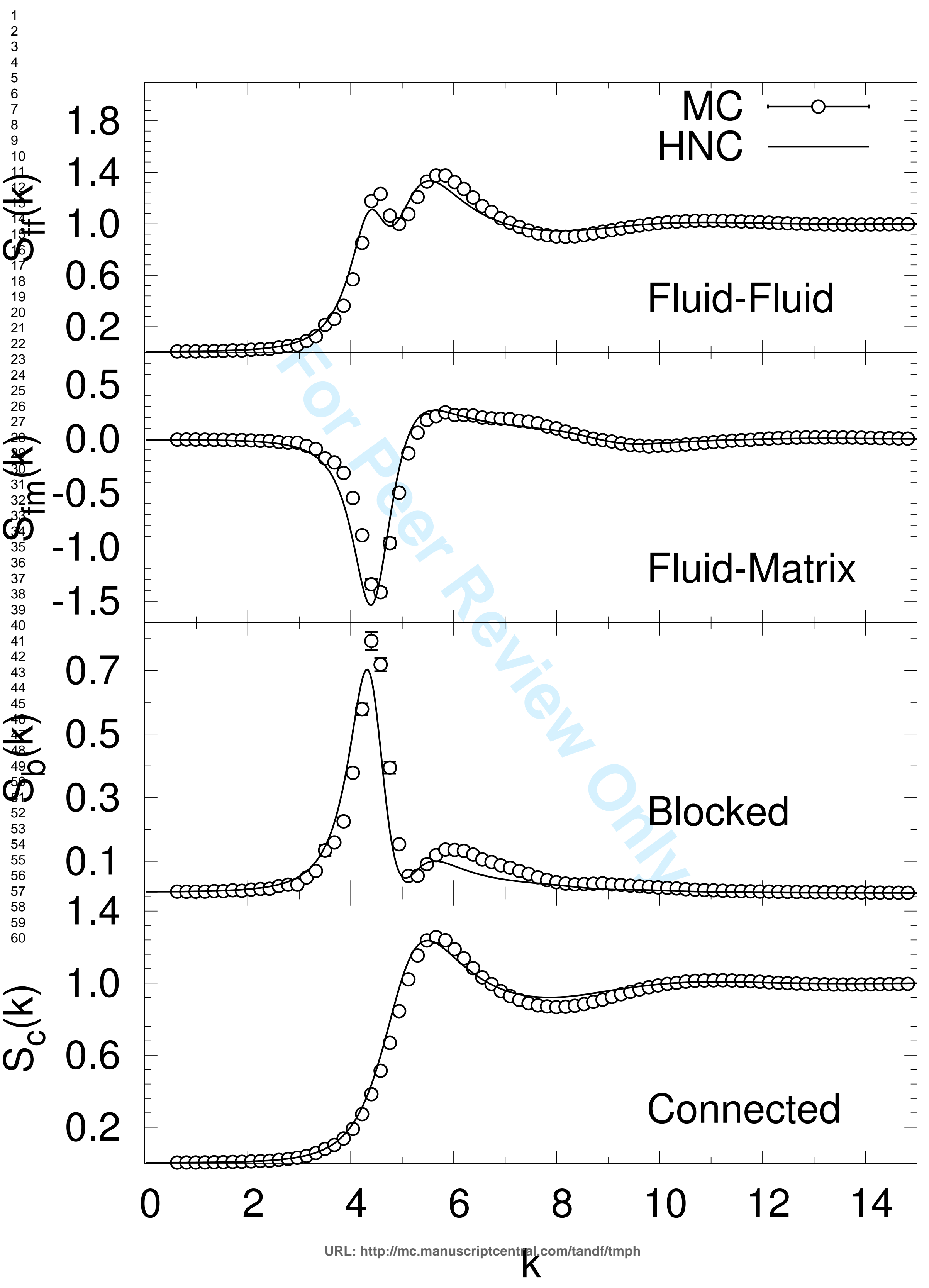




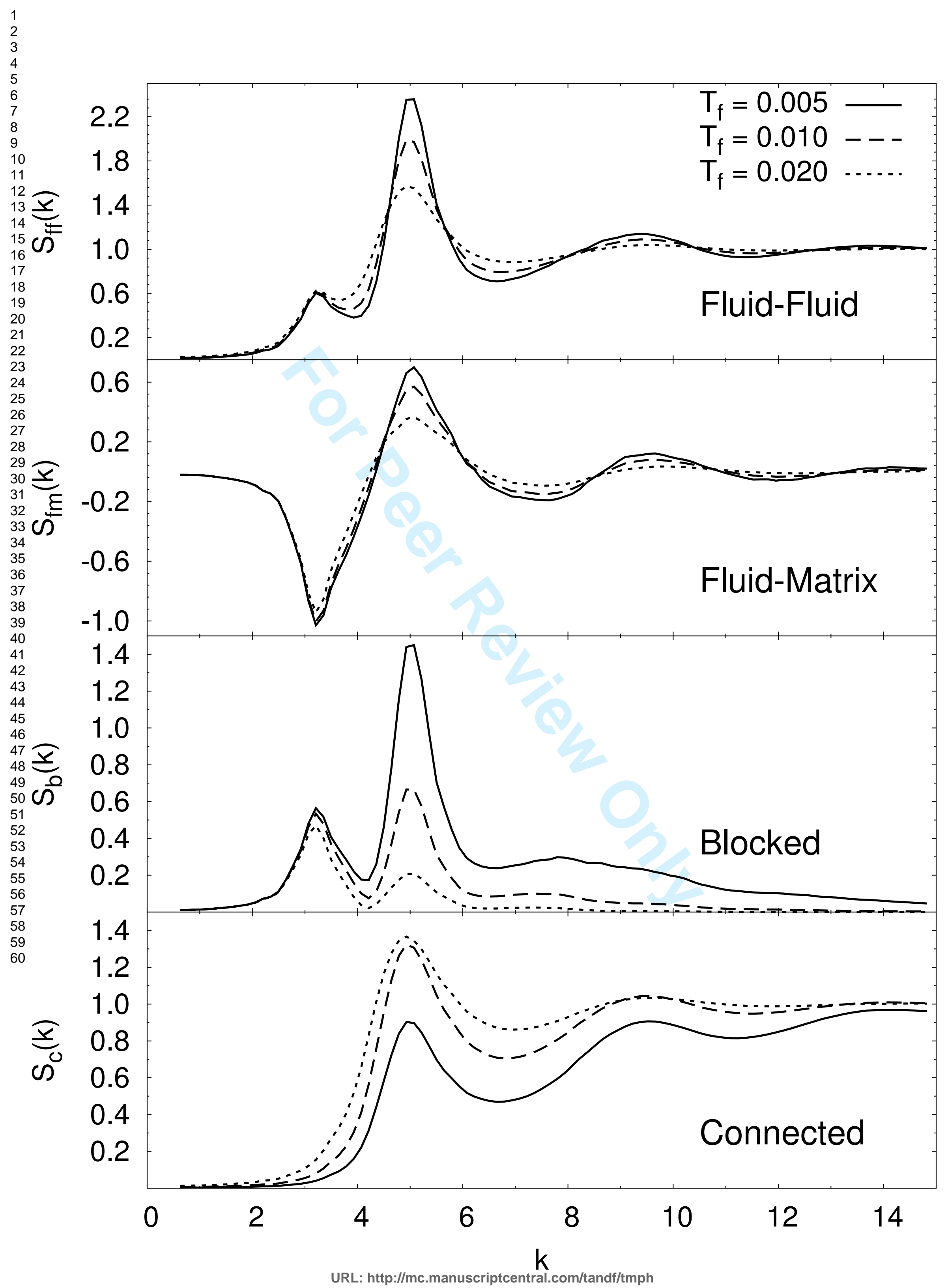




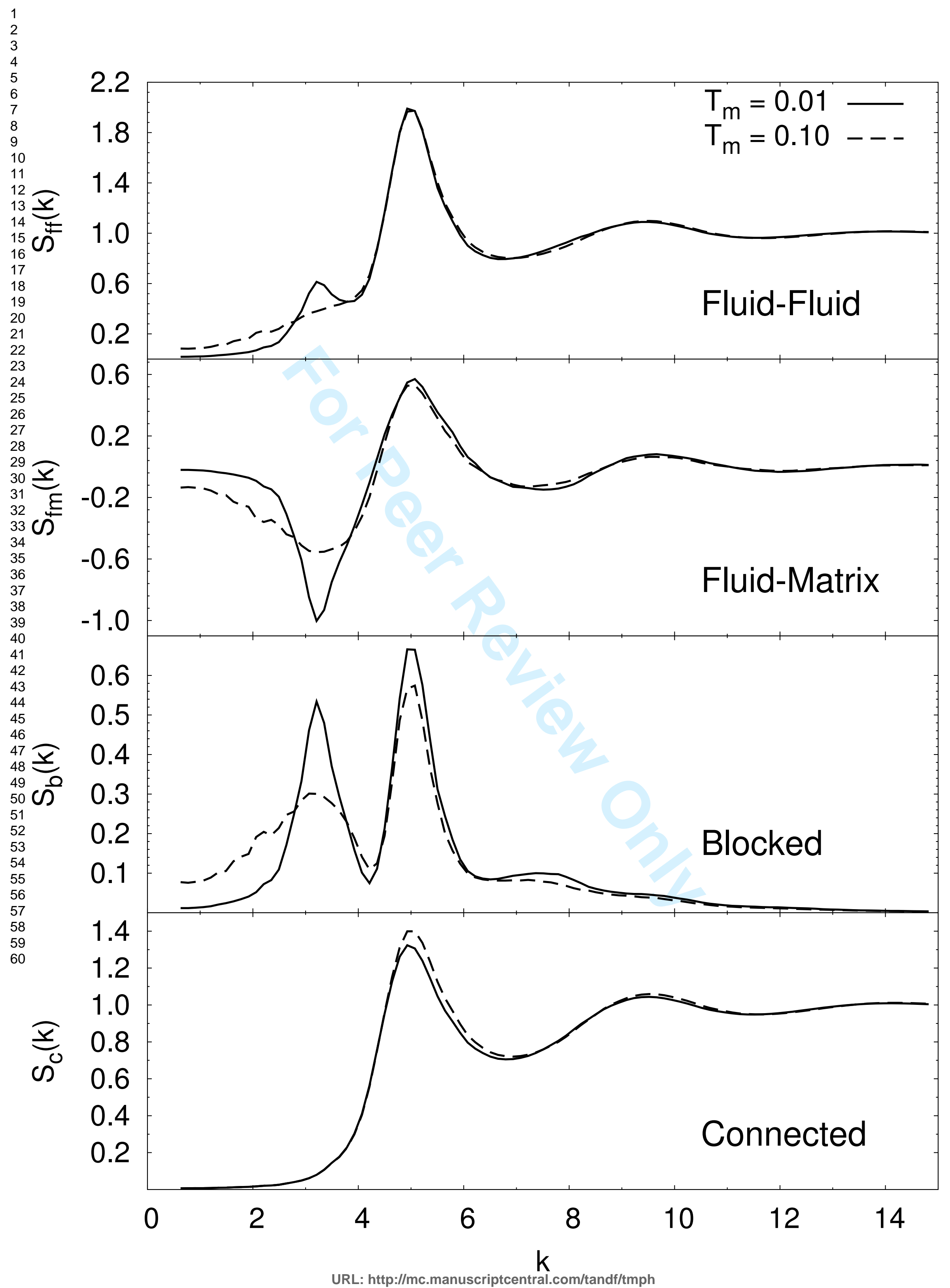




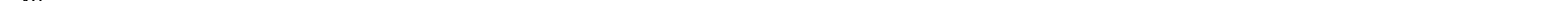

